\title{
One-year outcome of intravitreal aflibercept injection for age-related macular degeneration resistant to ranibizumab: rapid morphologic recovery and subsequent visual improvement
}

\author{
This article was published in the following Dove Press journal: \\ Clinical Ophthalmology \\ 26 May 2016 \\ Number of times this article has been viewed
}

\author{
Toshiaki Hirakata ${ }^{1,2, *}$ \\ Kaoru Fujinami ${ }^{1,3,4, *}$ \\ Ken Watanabe ${ }^{5}$ \\ Mariko Sasaki ${ }^{1,3,6}$ \\ Toru Noda ${ }^{1,5}$ \\ Kunihiko Akiyama ${ }^{1,5}$ \\ 'Laboratory of Visual Physiology, \\ Division for Vision Research, National \\ Institute of Sensory Organs, National \\ Hospital Organization, Tokyo Medical \\ Center, Tokyo, Japan; ${ }^{2}$ Department \\ of Ophthalmology, Juntendo \\ University of Graduate School of \\ Medicine, Tokyo, Japan; ${ }^{3}$ Department \\ of Ophthalmology, Keio University \\ School of Medicine, Tokyo, Japan; ${ }^{4} \mathrm{UCL}$ \\ Institute of Ophthalmology, London, \\ UK; ${ }^{5}$ Department of Ophthalmology, \\ National Hospital Organization, \\ Tokyo Medical Center, Tokyo, Japan; \\ ${ }^{6}$ Department of Ophthalmology, \\ Tachikawa Hospital, Tachikawa, \\ Tokyo, Japan \\ *These authors contributed equally \\ to this work
}

Objective: To describe the 1-year efficacy of aflibercept in Japanese patients with age-related macular degeneration (AMD) who were resistant to ranibizumab treatment.

Design: Retrospective case series.

Participants: Fourteen consecutive eyes of 14 patients with AMD were enrolled who had no substantial response or developed resistance to intravitreal ranibizumab injections.

Methods: All patients were subcategorized into one of two subtypes of AMD: seven patients with occult choroidal neovascularization (CNV) and seven with polypoidal choroidal vasculopathy (PCV). Serial intravitreal aflibercept (IVA) injections were administered. Comprehensive ophthalmic examinations, including optical coherence tomography, were conducted at baseline and at follow-up examinations at 1, 3, 6, and 12 months after the initial IVA injection. The bestcorrected visual acuity converted to logarithm of the minimum angle of resolution (logMAR) and central macular thickness (CMT) at each follow-up visit were compared with the baseline values. The anatomic response was also assessed with absorption or reduction of fluid in the subretina or subretinal pigment epithelial space.

Results: The logMAR best-corrected visual acuity improved significantly at 3, 6, and 12 months in the total cohort: at 3 and 6 months in patients with occult CNV and at 3 and 12 months in patients with PCV. The CMT decreased significantly at all follow-up visits in the total cohort as well as in both subtypes, except for the CMT at 6 months in PCV patients. The anatomic improvement was also demonstrated in all cases, and pigment epithelial detachments tended to be resolved more rapidly in patients with PCV than in patients with occult CNV.

Conclusion: Conversion to IVA was effective in patients with AMD resistant to ranibizumab, showing rapid morphologic improvement. The logMAR visual acuity was raised significantly within 12 months, and the clinical course of visual acuity improvement may differ according to the AMD subtypes.

Keywords: aflibercept, age-related macular degeneration, occult choroidal neovascularization, polypoidal choroidal vasculopathy, ranibizumab, resistance

\section{Introduction}

Department of Ophthalmology, National Institute of Sensory Organs, National Hospital Organization, Tokyo Medical Center, 2-5-I Higashigaoka, Meguro-ku,

Tokyo 152-8902, Japan

$\mathrm{Tel}+8 \mathrm{I} 334 \mathrm{II} 0$ III

Fax +8I 33412 98II

Email kakiyama@ntmc-hosp.jp
The prognosis of exudative age-related macular degeneration (AMD) has been improved significantly since anti-vascular endothelial growth factor (VEGF) therapy was introduced. ${ }^{1}$ The efficacy of anti-VEGF treatment has been established in various clinical trials using ranibizumab (Lucentis; Genentech Inc., South San Francisco, CA, USA) or bevacizumab (Avastin; Genentech Inc.); ${ }^{2-4}$ however, a certain proportion 
of patients with AMD have an inadequate or unsustained response to these drugs. ${ }^{5-7}$ For patients resistant to anti-VEGF therapy, injections of increased dose of anti-VEGF agents or combination therapy with other treatment options have been tried..$^{5,-11}$

Aflibercept (Eylea; Regeneron Pharmaceuticals, Tarrytown, NY, USA), a novel VEGF inhibitor with a high affinity for VEGF, ${ }^{12-14}$ has been introduced recently into clinical practice. Because of a wider range of pharmacological targets of aflibercept compared to ranibizumab or bevacizumab, switching to aflibercept has been considered a reasonable strategy in patients with AMD resistant to ranibizumab or bevacizumab, ${ }^{15-24}$ while no significant efficacy of conversion from ranibizumab to aflibercept was suggested in another study. ${ }^{25}$

Herein, we report the 1-year efficacy of conversion therapy to aflibercept in Japanese patients with AMD resistant to ranibizumab.

\section{Methods}

Fourteen eyes of 14 patients with neovascular AMD who had no significant response or developed resistance to intravitreal ranibizumab (IVR) injections were identified at the National Hospital Organization Tokyo Medical Center over a 12-month period. No significant response and resistance were defined, respectively, as no morphologic change after at least three IVR injections and an initial response to IVR injections with later recurrence and lack of morphologic improvement despite further repetitive IVR injections. The study protocol adhered to the provisions of the Declaration of Helsinki and was approved by the local Ethics Committee of the Tokyo Medical Center. Clinical trial registration number: R14-133.

After obtaining written informed consent, comprehensive ophthalmic examinations were performed in all 14 patients, including measurement of the decimal visual acuity (VA), fundus photography, fluorescein angiography (FA), indocyanine green angiography (ICGA), and spectral domain optical coherence tomography (SD-OCT) using the Cirrus HD-OCT system (Carl Zeiss Meditec, Dublin, CA, USA). The decimal VA was converted to the logarithm of the minimum angle of resolution ( $\log$ MAR) for statistical analysis. OCT images were acquired using the $512 \times 128$ macular cube protocol covering a $6 \times 6 \mathrm{~mm}$ area and/or the high-resolution five-line raster protocol with $9 \times 6 \mathrm{~mm}$ scans separated by $75 \mu \mathrm{m}$.

The diagnosis of AMD was confirmed by FA and ICGA at baseline. Subtypes of AMD were identified based on the FA or ICGA findings, and polypoidal choroidal vasculopathy
(PCV) and retinal angiomatous proliferation (RAP) were distinguished from a typical form of AMD. All 14 patients subsequently received an initial intravitreal aflibercept (IVA) injection from February 2013 to July 2013 followed by sequential injections according to monthly or treat-andextend regimens. ${ }^{26-28}$

To assess the efficacy of IVA treatment, two clinical parameters at 1, 3, 6, and 12 months after the first IVA injection were compared with the baseline values: the bestcorrected logMAR VA (BCVA) and the central macular thickness (CMT). The CMT was derived from the macular cube scans using the intrinsic automated software after adjustment of the fovea to the center, where needed. An anatomical response was assessed on OCT images by absorption or reduction of fluid at the subretina or subretinal pigment epithelial space using either the macular cube or the fiveline raster protocol. Assessment of anatomical response was conducted by two independent investigators (TH and KA), and only agreed data were used for further analysis.

Statistical analysis was performed with the Wilcoxon signed-rank test using IBM-SPSS software version 23.0 (IBM-SPSS, Inc., Chicago, IL, USA). Statistical significance was defined as $P<0.05$.

\section{Results}

The demographic data obtained from the 14 patients (nine men, five women) with AMD are presented in Table 1. Seven patients were subcategorized into occult choroidal neovascularization (CNV) and seven subjects into PCV. There were no cases of classic CNV or RAP. Two representative cases with marked improvement after IVA treatment are shown in Figures 1 (patient 3 with occult CNV) and 2 (patient 8 with PCV). The mean age of patients at baseline ( \pm standard deviation) was $77.7 \pm 9.4$ years. Seven patients were subcategorized into occult $\mathrm{CNV}$ and seven subjects into PCV. The data at 6 months were unavailable in patients 7 and 11 because the follow-up examinations were canceled by the patients. The average number of previous IVR injections was $12.4 \pm 10.0$, and the average number of total IVA injections was $7.7 \pm 2.3$. All the injections were performed properly, and no ocular or systemic adverse events were observed during the follow-up period.

The changes of BCVA are listed in Table 2. The mean BCVA of the 14 patients at baseline, 1, 3, 6, and 12 months follow-up was $0.40 \pm 0.27,0.39 \pm 0.28,0.29 \pm 0.27,0.26 \pm 0.21$, and $0.34 \pm 0.32$, respectively (Figure 3 ). The change from baseline reached statistical significance $(P=0.209, P=0.002$, $P=0.006$, and $P=0.018$ at $1,3,6$, and 12 months, respectively) 
Table I Demographics of 14 patients with AMD

\begin{tabular}{|c|c|c|c|c|c|c|}
\hline Patient number & Age & Sex & Eye & AMD subtype & $\begin{array}{l}\text { Number of previous } \\
\text { IVR injections }\end{array}$ & $\begin{array}{l}\text { Number of IVA injections } \\
\text { over I } 2 \text { months }\end{array}$ \\
\hline I & 82 & $M$ & $\mathrm{R}$ & Occult CNV & 4 & 6 \\
\hline 2 & 70 & $M$ & $\mathrm{R}$ & Occult CNV & 12 & 8 \\
\hline 3 & 66 & $M$ & L & Occult CNV & 9 & 8 \\
\hline 4 & 83 & $M$ & $\mathrm{R}$ & Occult CNV & 9 & 7 \\
\hline 5 & 95 & $\mathrm{~F}$ & L & Occult CNV & 5 & 11 \\
\hline 6 & 83 & $\mathrm{~F}$ & L & Occult CNV & 6 & 7 \\
\hline 7 & 96 & $M$ & L & Occult CNV & 10 & 6 \\
\hline 8 & 72 & $M$ & $\mathrm{R}$ & PCV & 27 & 5 \\
\hline 9 & 76 & $\mathrm{~F}$ & L & PCV & 6 & 7 \\
\hline 10 & 70 & $M$ & $\mathrm{R}$ & PCV & 10 & 10 \\
\hline II & 77 & $\mathrm{~F}$ & $\mathrm{R}$ & PCV & 12 & 4 \\
\hline 12 & 66 & $\mathrm{~F}$ & L & PCV & 41 & 12 \\
\hline 13 & 74 & $M$ & $\mathrm{R}$ & PCV & 15 & 7 \\
\hline 14 & 78 & $M$ & $\mathrm{R}$ & PCV & 7 & 10 \\
\hline Mean \pm SD & $77.7 \pm 9.4$ & & & & $12.4 \pm 10.0$ & $7.7 \pm 2.3$ \\
\hline
\end{tabular}

Abbreviations: AMD, age-related macular degeneration; CNV, choroidal neovascularization; IVA, intravitreal aflibercept; IVR, intravitreal ranibizumab; PCV, polypoidal choroidal vasculopathy; SD, standard deviation; L, left; R, right; M, male; F, female.

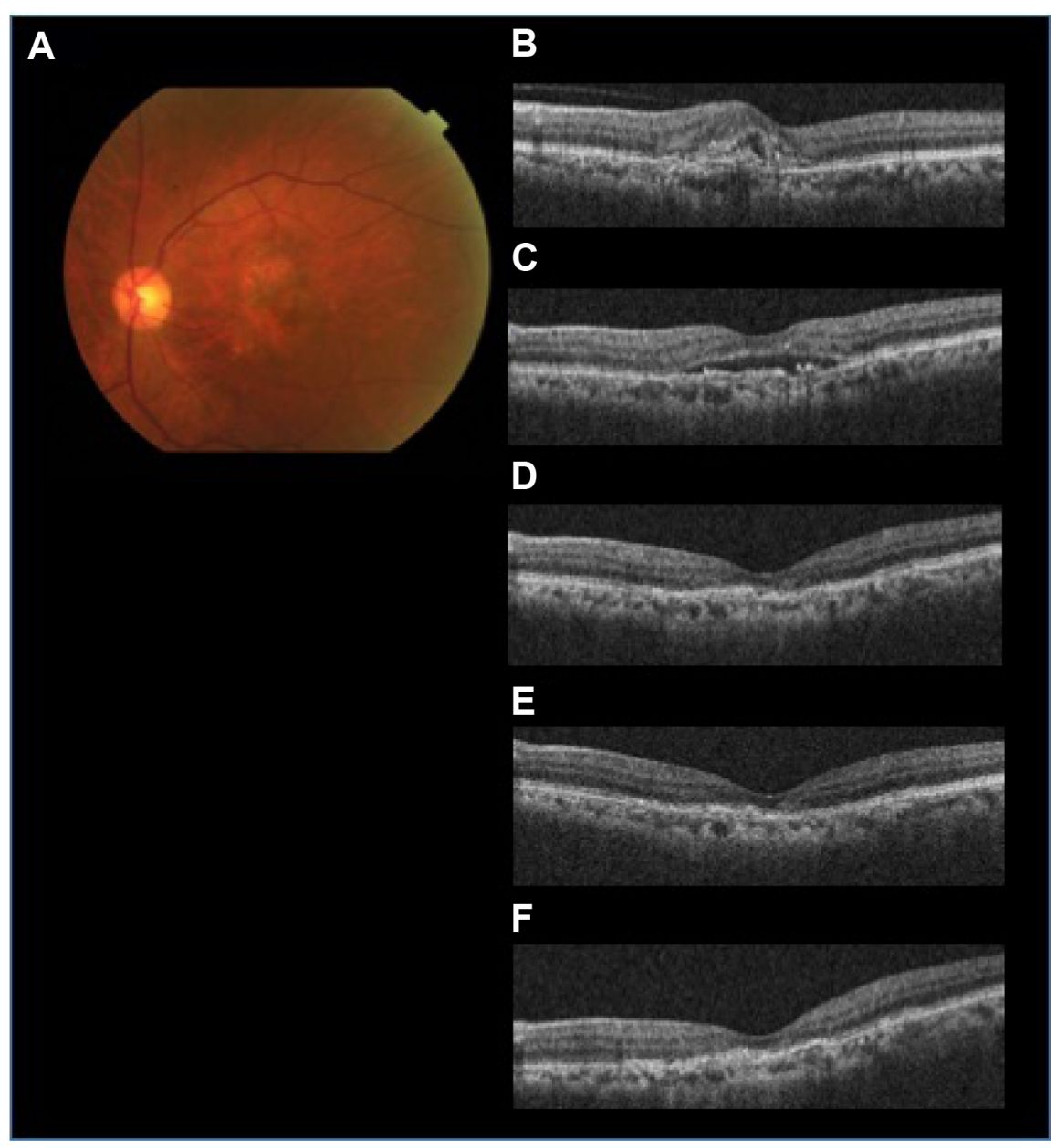

Figure I The efficacy of aflibercept for treating AMD resistant to ranibizumab (patient 3 with occult CNV).

Notes: A fundus photograph at baseline (A) and SD-OCT images prior to the initial IVR injection (B), at baseline (C), I month after the initial IVA injection (D), 3 months after the initial IVA injection (E), and I2 months after the initial IVA injection (F) in a 66-year-old man with occult CNV are shown. Marked SRF and a slight PED have improved at I-month follow-up examination, and the retinal structure has been maintained over 12 months.

Abbreviations: AMD, age-related macular degeneration; CNV, choroidal neovascularization; IVA, intravitreal aflibercept; IVR, intravitreal ranibizumab; PED, pigment epithelial detachment; SD-OCT, spectral domain optical coherence tomography; SRF, subretinal fluid. 


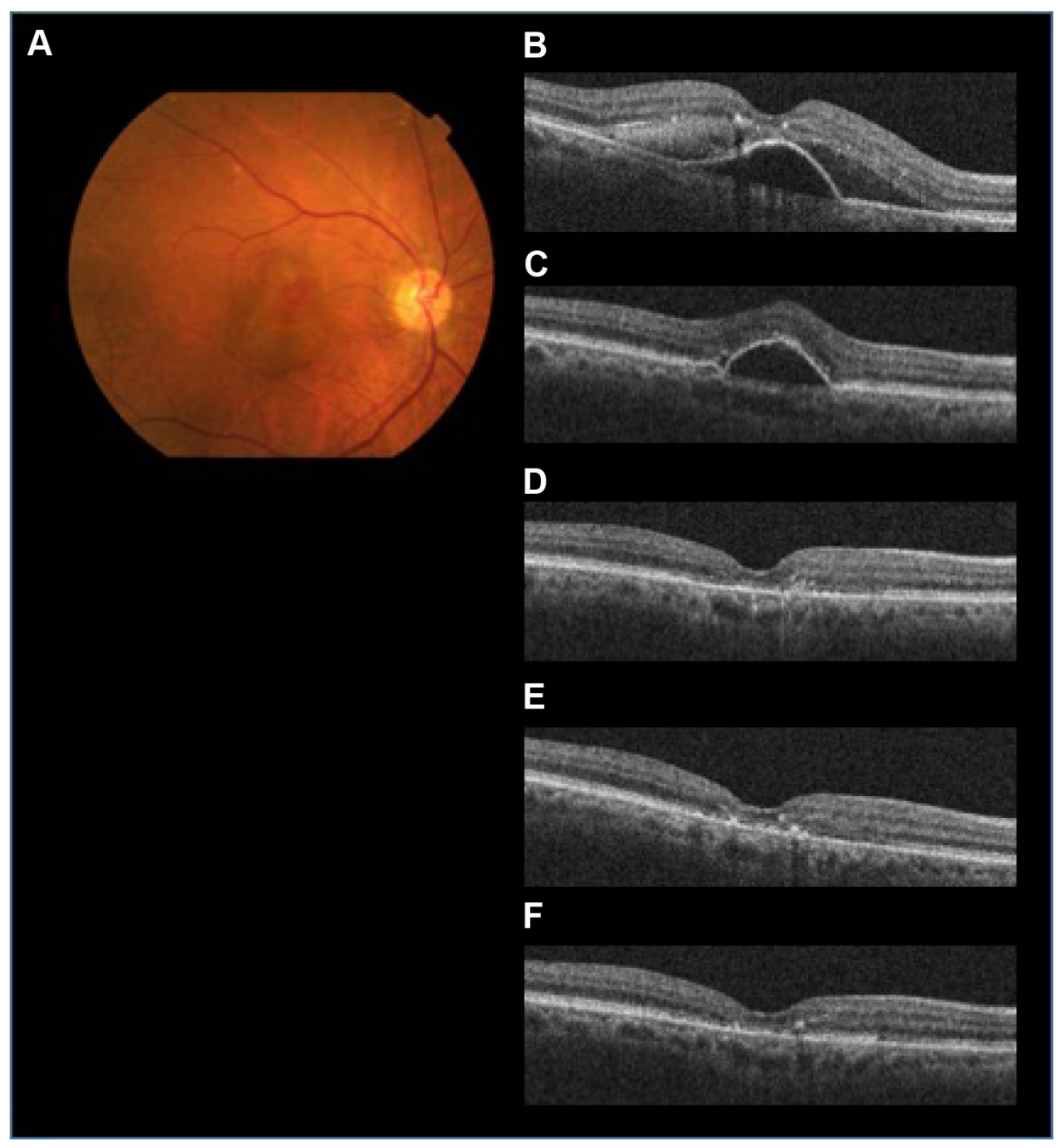

Figure 2 The efficacy of aflibercept for AMD refractory to ranibizumab (patient 8 with PCV).

Notes: A fundus photograph at baseline (A) and SD-OCT images prior to the initial IVR injection (B), at baseline (C), I month after the initial IVA injection (D), 3 months after the initial IVA injection (E), and 12 months after the initial IVA injection (F) in a 72-year-old man with PCV are shown. Slight SRF and prominent subfoveal PED have resolved completely at I-month follow-up examination, and the improved anatomic condition has been maintained over 12 months.

Abbreviations: AMD, age-related macular degeneration; IVA, intravitreal aflibercept; IVR, intravitreal ranibizumab; PCV, polypoidal choroidal vasculopathy; PED, pigment epithelial detachment; SD-OCT, spectral domain optical coherence tomography; SRF, subretinal fluid.

at the follow-up examinations of 3,6 , and 12 months. No significant difference was observed between baseline and 1-month follow-up (Figure 3). The mean BCVA of the seven patients with occult CNV improved significantly at 3 and 6 months ( $P=0.028$ and $P=0.027$, respectively) (Figure 3), whereas the mean BCVA of the seven PCV patients improved significantly only at 3 and 12 months $(P=0.028$ and $P=0.027$, respectively) (Figure 3).

The CMT changes are shown in Table 3. The CMT measurements were unavailable for patients $2,7,9,10$, and 11 at some visits because of the low image quality or the inappropriate recording protocol. The average CMT of the 14 patients at baseline and at 1, 3,6, and 12 months follow-up was $273.2 \pm 65.2,209.1 \pm 42.0,215.0 \pm 46.4,227.4 \pm 36.3$, and $208.2 \pm 38.2 \mu \mathrm{m}$, respectively (Figure 3). Significant reduction of CMT compared with baseline was revealed $(P=0.002$, $P=0.003, P=0.028$, and $P=0.002$, respectively) at all follow-up examinations (Figure 3 ). In the seven patients with occult AMD, the CMT at each examination decreased significantly from baseline $(303.1 \pm 59.0 \mu \mathrm{m})$ to 1 month $(232.4 \pm 42.1 \mu \mathrm{m})$, 3 months $(225.0 \pm 45.4 \mu \mathrm{m}), 6$ months $(253.2 \pm 21.1 \mu \mathrm{m})$, and 12 months $(220.2 \pm 33.6 \mu \mathrm{m})$ (Figure 3$)$. Significant decrease was found at each time point $(P=0.028, P=0.028, P=0.043$, and $P=0.028$, respectively). The CMT of patients with PCV also decreased compared with baseline $(238.2 \pm 57.5 \mu \mathrm{m})$ at 1 month $(181.8 \pm 21.1 \mu \mathrm{m}), 3$ months $(201.0 \pm 49.0 \mu \mathrm{m})$, 6 months $(201.6 \pm 29.3 \mu \mathrm{m})$, and 12 months $(196.2 \pm 41.7 \mu \mathrm{m})$. Significant reduction of CMT was revealed at 1, 3, and 12 months ( $P=0.027, P=0.043$, and $P=0.028$, respectively). The value at 6 months did not reach significance $(P=0.345)$ 
Table 2 Best-corrected visual acuity of I4 patients treated with IVA injection

\begin{tabular}{|c|c|c|c|c|c|c|}
\hline \multirow[t]{2}{*}{ Patient number } & \multirow[t]{2}{*}{ AMD subtype } & \multicolumn{5}{|c|}{ BCVA (logMAR) } \\
\hline & & Baseline & I month & 3 months & 6 months & 12 months \\
\hline I & Occult CNV & 0.52 & 0.52 & 0.40 & 0.40 & 0.30 \\
\hline 2 & Occult CNV & 0.52 & 0.70 & 0.52 & 0.40 & 0.52 \\
\hline 3 & Occult CNV & 0.40 & 0.40 & 0.40 & 0.40 & 0.40 \\
\hline 4 & Occult CNV & 0.22 & 0.10 & 0.00 & 0.15 & 0.30 \\
\hline 5 & Occult CNV & 0.52 & 0.52 & 0.30 & 0.22 & 0.40 \\
\hline 6 & Occult CNV & 0.40 & 0.30 & 0.30 & 0.22 & 0.40 \\
\hline 7 & Occult CNV & 1.00 & 1.00 & 0.82 & NA & 1.10 \\
\hline Mean \pm SD $(P t ~ I-7)$ & & $0.5 I \pm 0.24$ & $0.50 \pm 0.29$ & $0.39 \pm 0.25$ & $0.30 \pm 0.11$ & $0.49 \pm 0.28$ \\
\hline$P$-value & & & $P=0.462$ & $P=0.028^{*}$ & $P=0.027^{*}$ & $P=0.397$ \\
\hline 8 & PCV & 0.15 & 0.10 & 0.05 & 0.22 & 0.00 \\
\hline 9 & PCV & 0.15 & 0.15 & 0.00 & 0.05 & 0.00 \\
\hline 10 & $\mathrm{PCV}$ & 0.05 & 0.05 & 0.00 & -0.08 & -0.08 \\
\hline II & $\mathrm{PCV}$ & 0.30 & 0.22 & 0.15 & NA & 0.15 \\
\hline 12 & PCV & 0.40 & 0.52 & 0.40 & 0.40 & 0.52 \\
\hline 13 & PCV & 0.82 & 0.70 & 0.70 & 0.70 & 0.70 \\
\hline 14 & PCV & 0.15 & 0.15 & 0.00 & 0.10 & 0.00 \\
\hline Mean \pm SD $(P t$ 8-14) & & $0.29 \pm 0.26$ & $0.27 \pm 0.24$ & $0.19 \pm 0.27$ & $0.23 \pm 0.28$ & $0.18 \pm 0.30$ \\
\hline$P$-value & & & $P=0.248$ & $P=0.028^{*}$ & $P=0.116$ & $P=0.027^{*}$ \\
\hline Mean \pm SD $(P t ~ I-14)$ & & $0.40 \pm 0.27$ & $0.39 \pm 0.28$ & $0.29 \pm 0.27$ & $0.26 \pm 0.21$ & $0.34 \pm 0.32$ \\
\hline$P$-value & & & $P=0.209$ & $P=0.002^{*}$ & $P=0.006^{*}$ & $P=0.018^{*}$ \\
\hline
\end{tabular}

Notes: Statistical analysis was performed with the Wilcoxon signed-rank test. *Significant difference was defined as $P<0.05$ comparing BCVA (logMAR) at each visit with baseline.

Abbreviations: AMD, age-related macular degeneration; BCVA, best-corrected visual acuity; CNV, choroidal neovascularization; IVA, intravitreal aflibercept; logMAR, logarithm of the minimum angle of resolution; NA, not applicable; PCV, polypoidal choroidal vasculopathy; Pt, patient; SD, standard deviation.

(Figure 3). The CMT of patients 8 and 12 showed a different trend; increased from 214 and $184 \mu \mathrm{m}$ at baseline to 220 and $213 \mu \mathrm{m}$ at 6 months, respectively.

Anatomic improvement was confirmed in all patients (Table 4). Subretinal fluid (SRF) was observed in eleven patients at baseline and resolved completely at 1 month in eight patients, at 3 months in one patient, and at 6 months in one patient. OCT showed complete resolution of SRF within 6 months in all six patients with occult CNV and in four of five patients with PCV who had SRF at baseline. A pigment epithelial detachment (PED) was present at baseline in all 14 patients and resolved completely at 1 month in six patients (two patients with occult CNV, four patients with PCV) followed by later complete regression in two patients
A

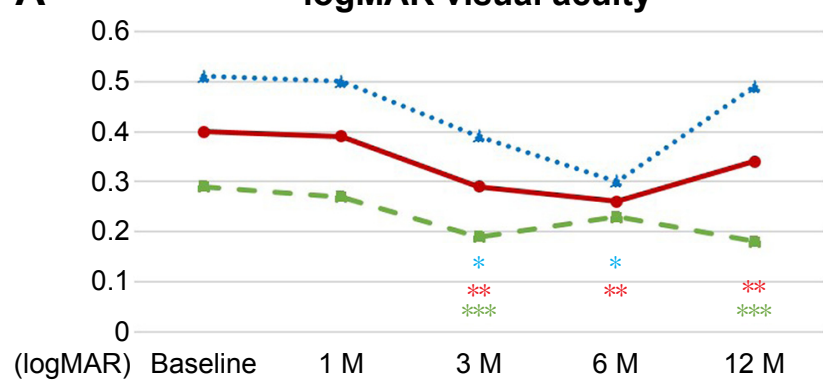

B

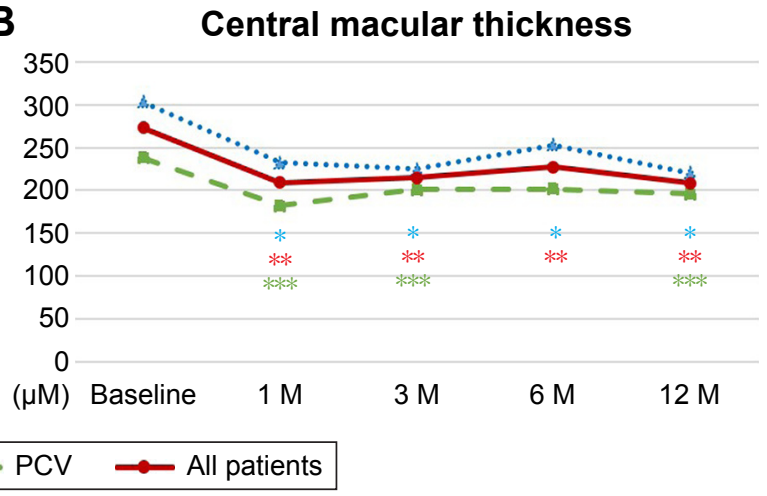

Figure 3 The efficacy of aflibercept assessed based on the visual acuity and central macular thickness in I4 patients with AMD (seven patients with occult CNV and seven subjects with PCV).

Notes: The efficacy of aflibercept is assessed based on visual acuity converted to logMAR (A) and the central macular thickness measured by SD-OCT (B). Data obtained at I month (I M), 3 months (3 M), 6 months (6 M), and 12 months (I2 M) after the first IVA injection are compared with baseline data. Subcategorical data based on the subtype classifications are also demonstrated (seven patients with occult CNV and seven subjects with PCV). The line charts of the mean data for occult CNV, PCV, and the total cohort are shown in different colors. Statistical significance was defined as $P<0.05$. Asterisks indicate significant differences (Wilcoxon signed-rank test, $*=0 c c u l t$ CNV, **=PCV, ****=total cohort).

Abbreviations: AMD, age-related macular degeneration; CNV, choroidal neovascularization; IVA, intravitreal aflibercept; logMAR, logarithm of minimum angle of resolution; PCV, polypoidal choroidal vasculopathy; SD-OCT, spectral domain optical coherence tomography. 
Table 3 Central macular thickness of 14 patients treated with IVA

\begin{tabular}{|c|c|c|c|c|c|c|}
\hline \multirow[t]{2}{*}{ Patient number } & \multirow[t]{2}{*}{ AMD subtype } & \multicolumn{5}{|l|}{ CMT $(\mu \mathrm{m})$} \\
\hline & & Baseline & I month & 3 months & 6 months & 12 months \\
\hline I & Occult CNV & 330 & 222 & 226 & 225 & 225 \\
\hline 2 & Occult CNV & 397 & 214 & 185 & NA & NA \\
\hline 3 & Occult CNV & 315 & 185 & 180 & 259 & 181 \\
\hline 4 & Occult CNV & 297 & 306 & 305 & 265 & 251 \\
\hline 5 & Occult CNV & 245 & 226 & 217 & 239 & 219 \\
\hline 6 & Occult CNV & 322 & 272 & 264 & 278 & 262 \\
\hline 7 & Occult CNV & 219 & 202 & 198 & NA & 183 \\
\hline Mean \pm SD $($ Pt I-7) & & $303.1 \pm 59.0$ & $232.4 \pm 42.1$ & $225.0 \pm 45.4$ & $253.2 \pm 21.1$ & $220.2 \pm 33.6$ \\
\hline$P$-value & & & $P=0.028^{*}$ & $P=0.028^{*}$ & $P=0.043^{*}$ & $P=0.028^{*}$ \\
\hline 8 & PCV & 214 & 194 & 186 & 220 & 187 \\
\hline 9 & PCV & 246 & 170 & NA & 202 & 174 \\
\hline 10 & PCV & NA & NA & NA & NA & NA \\
\hline II & PCV & 304 & 210 & 207 & NA & 250 \\
\hline 12 & PCV & 184 & 165 & 175 & 213 & 179 \\
\hline 13 & PCV & 175 & 156 & 155 & $|5|$ & 144 \\
\hline 14 & PCV & 306 & 196 & 282 & 222 & 243 \\
\hline Mean \pm SD $(P t$ 8-14) & & $238.2 \pm 57.5$ & $|8| .8 \pm 21 . \mid$ & $20 I .0 \pm 49.0$ & $201.6 \pm 29.3$ & $|96.2 \pm 4| .7$ \\
\hline$P$-value & & & $P=0.027^{*}$ & $P=0.043^{*}$ & $P=0.345$ & $P=0.028^{*}$ \\
\hline Mean \pm SD $(\mathrm{Pt} \mathrm{I}-14)$ & & $273.2 \pm 65.2$ & $209.1 \pm 42.0$ & $215.0 \pm 46.4$ & $227.4 \pm 36.3$ & $208.2 \pm 38.2$ \\
\hline$P$-value & & & $P=0.002 *$ & $P=0.003^{*}$ & $P=0.028^{*}$ & $P=0.002^{*}$ \\
\hline
\end{tabular}

Notes: Statistical analysis was performed with the Wilcoxon signed rank test. *Significant difference was defined as $P<0.05$ comparing $C M T$ at each visit with baseline. Abbreviations: AMD, age-related macular degeneration; CMT, central macular thickness; CNV, choroidal neovascularization; NA, not applicable; PCV, polypoidal choroidal vasculopathy; $\mathrm{Pt}$, patient; $\mathrm{SD}$, standard deviation.

with occult CNV at 3 and 12 months. Complete regression of the PED occurred in four of seven patients with occult CNV and in four of seven patients with PCV within 12 months. In six patients with persistent SRF and/or PED (patients 4, 5, 6, 10, 12, and 14), morphologic improvement was confirmed at the 1-month examination on the OCT images.

\section{Discussion}

Rapid morphologic improvement following treatment with aflibercept was shown in all 14 Japanese patients who were refractory to previous ranibizumab treatment. The efficacy of aflibercept was verified at the 1-month follow-up visit after the initial IVA injection and lasted for 12 months.

Table 4 Anatomical response to IVA treatment in 14 patients with AMD

\begin{tabular}{|c|c|c|c|c|c|c|c|c|c|c|c|c|c|}
\hline \multirow{2}{*}{$\begin{array}{l}\text { Patient } \\
\text { number }\end{array}$} & \multirow{2}{*}{ AMD subtype } & \multicolumn{2}{|c|}{ Baseline } & \multicolumn{2}{|c|}{ I month } & \multicolumn{2}{|c|}{3 months } & \multicolumn{2}{|c|}{6 months } & \multicolumn{2}{|c|}{12 months } & \multirow{2}{*}{$\begin{array}{l}\text { Complete resolution } \\
\text { of SRF/PED (month) }\end{array}$} & \multirow{2}{*}{$\begin{array}{l}\text { Anatomic improvement } \\
\text { assessed by OCT }\end{array}$} \\
\hline & & SRF & PED & SRF & PED & SRF & PED & SRF & PED & SRF & PED & & \\
\hline I & Occult CNV & ++ & ++ & - & - & - & - & - & - & - & - & $+/+(I / I)$ & + \\
\hline 2 & Occult CNV & ++ & ++ & + & - & - & + & - & - & - & - & $+/+(3 / I)$ & + \\
\hline 3 & Occult CNV & ++ & ++ & - & ++ & - & - & + & + & - & - & $+/+(1 / 3)$ & + \\
\hline 4 & Occult CNV & ++ & ++ & + & + & + & + & - & + & - & + & $+/-(6 /-)$ & + \\
\hline 5 & Occult CNV & - & ++ & - & + & - & + & + & + & - & + & $* /-$ & + \\
\hline 6 & Occult CNV & ++ & ++ & - & + & - & + & + & + & + & + & $+/-(1 /-)$ & + \\
\hline 7 & Occult CNV & ++ & ++ & - & + & - & + & NA & NA & - & - & $+/+(I / I 2)$ & + \\
\hline 8 & PCV & - & ++ & - & - & - & - & + & + & - & - & $* /+(* / I)$ & + \\
\hline 9 & PCV & ++ & ++ & - & - & - & - & + & + & - & - & $+/+(I / I)$ & + \\
\hline 10 & PCV & ++ & ++ & - & + & - & + & - & + & - & + & $+1-(1 /-)$ & + \\
\hline II & PCV & ++ & ++ & - & - & - & - & NA & NA & - & - & $+/+(I / I)$ & + \\
\hline 12 & PCV & - & ++ & - & + & - & + & - & + & - & + & $* /-$ & + \\
\hline 13 & PCV & ++ & ++ & - & - & - & - & - & - & - & - & $+/+(I / I)$ & + \\
\hline 14 & PCV & ++ & ++ & + & + & + & + & + & + & + & + & $-1-$ & + \\
\hline
\end{tabular}

Notes: Anatomic response was determined with spectral domain optical coherence tomography by assessing change in subretinal fluid or subretinal pigment epithelial fluid. SRF and PED observed by OCT: $++=$ detected at baseline/ $-=$ not detected at baseline; $++=$ no change, $+=$ improved (from ++ ) or newly appeared (from - ), $-=$ not detected at I, 3, 6 , and 12 months. Anatomic OCT change: $+=$ improved. Complete resolution of SRF/PED: $+=$ completely resolved, $-=$ not completely resolved, $*=$ not detectable at baseline. Abbreviations: AMD, age-related macular degeneration; CNV, choroidal neovascularization; IVA, intravitreal aflibercept; PCV, polypoidal choroidal vasculopathy; PED, pigment epithelial detachment; OCT, optical coherence tomography; SRF, subretinal fluid. 
Moreover, complete reduction of the PEDs, which never occurred with the IVR injections, was observed in eight of 14 patients with AMD.

There are at least two possible theories to explain the effectiveness of aflibercept in patients who are resistant to other anti-VEGF agents. The first is the difference in pharmacological targets, with aflibercept having a wider range of targets including VEGF-A, VEGF-B, and placental growth factor compared to ranibizumab (VEGF-A). ${ }^{14,20}$ The rapid morphologic improvement in the current study after switching to aflibercept may have resulted from the effect of IVA on the recalcitrant factors related to VEGF-B or placental growth factor, in addition to the well-inhibited exudative elements related to VEGF with pretreatment of IVR. These supplementary mechanisms of aflibercept may provide an immediate step forward to complete regression of the remaining exudation. Another plausible explanation relates to tachyphylaxis or tolerance, which can be addressed by switching drugs, drug cessation, and increasing dosage. .,16-20,29 $^{\text {, }}$

Regarding the clinical subtypes of AMD, PCV has been discussed as a sign of poor responders to ranibizumab. Although ranibizumab has been reported to be fairly effective for treating PCV, complete regression of polypoidal lesions can be expected in less than half of the patients receiving IVR injections..$^{30}$ On the other hand, resolution of polypoidal lesion by IVA treatment has been reported in $69.2 \%{ }^{31}$ and $55.4 \%{ }^{32}$ of cases with PCV in 12 months. Some investigators have mentioned aflibercept may benefit patients with PCV because aflibercept has a wide range of targets possibly critical in angiogenesis ${ }^{14}$ and related to activities of PCV vascular lesions. ${ }^{18}$ This hypothesis supports the efficacy of aflibercept in all seven patients with PCV in the current study. Different demographic features such as a high rate of PCV have been well-known in Japanese AMD population compared with those in Caucasian population. ${ }^{26,33}$ Although the increased prevalence of PCV has also been reported in the Caucasian AMD patients with a poor response to ranibizumab, ${ }^{7}$ the ethnic background underlying the AMD subtypes is likely to be a key in prediction of the aflibercept efficacy for patients with refractory AMD.

PED is another factor associated with resistance to ranibizumab. Suzuki et $\mathrm{al}^{6}$ reported that fibrovascular PED and Type $1 \mathrm{CNV}$ were associated with nonresponders to IVR injections. Parodi et $\mathrm{al}^{34}$ also described persistent PEDs in $77.5 \%$ of patients with occult CNV after 24 months of IVR injections. In contrast, all seven patients with occult CNV in the current study had morphologic improvement, especially with absorption or reduction of subretinal pigment epithelial fluid and SRF. The PEDs regressed completely in four of the seven patients with occult CNV, and SRF was absorbed in all six patients with SRF at baseline. These results indicate that aflibercept is also effective in occult CNV with PEDs that had a poor response to ranibizumab. Furthermore, the more rapid $\mathrm{PED}$ resolution was also suggested in patients with $\mathrm{PCV}$ than in patients with occult CNV in the current study. This finding might be explained with the incorporative effect on PED by IVA following IVR, as already discussed.

Interestingly, the course of VA improvement in seven patients with occult $\mathrm{CNV}$ was not parallel to the course of CMT changes. The recovered VA was not maintained at 12 months, whereas the CMT showed improved retinal structure continuously toward the 12-month visit. Various courses after switching to IVA could be explained by the different characteristics of vasculature between these two subtypes, although it is difficult to determine the difference of visual prognosis between occult CNV and PCV, considering the small sample size of the current study. ${ }^{35-37}$ Previous studies have reported two different visual prognoses in recalcitrant cases converted to IVA injections; some showed improvement and in others the VA was maintained after treatment. ${ }^{15,16,19,20,23}$ Several attributable factors have been discussed regarding this discrepancy in visual outcomes: the length of follow-up, distribution of AMD subtypes, therapy protocols, and study designs. ${ }^{15,20,25}$ As far as the current study goes, there were advantages possibly affecting the visual outcome; the12-month follow-up (adequate to observe the visual improvement); ${ }^{16}$ the suitable AMD subtypes (confirmed by FA and ICGA); and the smaller number of previous IVR injections (suggesting less damage to the macular function at baseline). ${ }^{15,16,19,20}$

Only a few reports provide the visual outcome of the conversion therapy in a prospective study design. Mantel et $\mathrm{al}^{25}$ reported in a prospective study that conversion to IVA in recalcitrant patients showed similar morphologic and visual outcomes compared to continuous monthly IVR injections. In addition, Kawashima et $\mathrm{al}^{23}$ mentioned better visual prognosis in recalcitrant patients with PCV than typical occult CNV after conversion to IVA in a prospective study design. Given the fact that subcategorization of AMD subtypes was not described in Mantel's report, the distribution of the AMD subtypes may determine the treatment outcomes. In other words, the proportion of PCV in a cohort could be related to the favorable outcome of the conversion therapy. More numbers of prospective studies in large cohorts are needed to find out the prognostic factors in the conversion therapy.

There are limitations in the current study. The number of cases was relatively small, with some lacking data for the subtype analysis. The treatment protocol was not perfectly 
standardized in all cases. Therefore, a larger cohort with longterm follow-up over 12 months could provide more powerful evidence of the different efficacy of IVR or IVA and visual improvement after conversion to IVA in refractory AMD.

In summary, the rapid response to aflibercept in patients with AMD resistant to ranibizumab has been supported by morphologic improvement, and the effect lasted for 12 months. PEDs tended to be resolved more rapidly in patients with PCV than in patients with occult CNV in the current study. Visual improvement can be predicted within 12 months from conversion to IVA injections, and further investigation is needed to elucidate the desirable protocol of the conversion strategy for refractory AMD.

\section{Acknowledgments}

Kunihiko Akiyama has received lecture fees from Santen Pharmaceutical Co., Ltd (Osaka, Japan) and consultancy fees from Bayer Yakuhin, Ltd. (Osaka, Japan). No other authors have any financial interest in any product mentioned in this report.

This research received no specific grant from any funding agency.

\section{Disclosure}

The author reports no conflicts of interest in this work.

\section{References}

1. Lanzetta P, Mitchell P, Wolf S, Veritti D. Different antivascular endothelial growth factor treatments and regimens and their outcomes in neovascular age-related macular degeneration: a literature review. Br J Ophthalmol. 2013;97(12):1497-1507.

2. Brown DM, Michels M, Kaiser PK, et al. Ranibizumab versus verteporfin photodynamic therapy for neovascular age-related macular degeneration: two-year results of the ANCHOR study. Ophthalmology. 2009; 116(1):57-65.e55.

3. Rosenfeld PJ, Brown DM, Heier JS, et al. Ranibizumab for neovascular age-related macular degeneration. $N$ Engl J Med. 2006;355(14): 1419-1431.

4. Comparison of Age-related Macular Degeneration Treatments Trials Research Group, Martin DF, Maguire MG, Fine SL, et al. Ranibizumab and bevacizumab for treatment of neovascular age-related macular degeneration: two-year results. Ophthalmology. 2012;119(7): $1388-1398$.

5. Wykoff CC, Brown DM, Croft DE, Wong TP. Two Year SAVE Outcomes: $2.0 \mathrm{mg}$ ranibizumab for recalcitrant neovascular AMD. Ophthalmology. 2013;120(9):1945-1946.e1941.

6. Suzuki M, Nagai N, Izumi-Nagai K, et al. Predictive factors for nonresponse to intravitreal ranibizumab treatment in age-related macular degeneration. Br J Ophthalmol. 2014;98(9):1186-1191.

7. Hatz K, Prunte C. Polypoidal choroidal vasculopathy in Caucasian patients with presumed neovascular age-related macular degeneration and poor ranibizumab response. Br J Ophthalmol. 2014;98(2): 188-194.

8. Calvo P, Ferreras A, Al Adel F, Wang Y, Brent MH. Dexamethasone intravitreal implant as adjunct therapy for patients with wet age-related macular degeneration with incomplete response to ranibizumab. Br J Ophthalmol. 2015;99(6):723-726.
9. Koh A, Lee W, Chen L, et al. EVEREST study: efficacy and safety of verteporfin photodynamic therapy in combination with ranibizumab or alone versus ranibizumab monotherapy in patients with symptomatic macular polypoidal choroidal vasculopathy. Retina. 2012; 32(8):1453-1464

10. Silva-Garcia R, McLellan C, Shaya FS, Small KW. Long-lasting effects of anti-VEGF/photodynamic combination therapy in the treatment of exudative age-related macular degeneration: a retrospective chart review. Clin Ophthalmol. 2014;8:2529-2532.

11. Sato T, Kishi S, Matsumoto H, Mukai R. Comparisons of outcomes with different intervals between adjunctive ranibizumab and photodynamic therapy for polypoidal choroidal vasculopathy. Am J Ophthalmol. 2013; 156(1):95-105.e101.

12. Stewart MW, Rosenfeld PJ. Predicted biological activity of intravitreal VEGF Trap. Br J Ophthalmol. 2008;92(5):667-668.

13. Fauser S, Schwabecker V, Muether PS. Suppression of intraocular vascular endothelial growth factor during aflibercept treatment of age-related macular degeneration. Am J Ophthalmol. 2014;158(3): 532-536.

14. Papadopoulos N, Martin J, Ruan Q, et al. Binding and neutralization of vascular endothelial growth factor (VEGF) and related ligands by VEGF Trap, ranibizumab and bevacizumab. Angiogenesis. 2012;15(2): 171-185.

15. Chang AA, Li H, Broadhead GK, et al. Intravitreal aflibercept for treatment-resistant neovascular age-related macular degeneration. Ophthalmology. 2014;121(1):188-192.

16. Kumar N, Marsiglia M, Mrejen S, et al. Visual and anatomical outcomes of intravitreal aflibercept in eyes with persistent subfoveal fluid despite previous treatments with ranibizumab in patients with neovascular agerelated macular degeneration. Retina. 2013;33(8):1605-1612.

17. Bakall B, Folk JC, Boldt HC, et al. Aflibercept therapy for exudative age-related macular degeneration resistant to bevacizumab and ranibizumab. Am J Ophthalmol. 2013;156(1):15-22.e11.

18. Miura M, Iwasaki T, Goto $\mathrm{H}$. Intravitreal aflibercept for polypoidal choroidal vasculopathy after developing ranibizumab tachyphylaxis. Clin Ophthalmol. 2013;7:1591-1595.

19. Wykoff CC, Brown DM, Maldonado ME, Croft DE. Aflibercept treatment for patients with exudative age-related macular degeneration who were incomplete responders to multiple ranibizumab injections (TURF trial). Br J Ophthalmol. 2014;98(7):951-955.

20. Yonekawa Y, Andreoli C, Miller JB, et al. Conversion to aflibercept for chronic refractory or recurrent neovascular age-related macular degeneration. Am J Ophthalmol. 2013;156(1):29-35.e22.

21. Grewal DS, Gill MK, Sarezky D, Lyon AT, Mirza RG. Visual and anatomical outcomes following intravitreal aflibercept in eyes with recalcitrant neovascular age-related macular degeneration: 12-month results. Eye (Lond). 2014;28(7):895-899.

22. Singh RP, Srivastava S, Ehlers JP, Bedi R, Schachat AP, Kaiser PK. A singlearm, investigator-initiated study of the efficacy, safety and tolerability of intravitreal aflibercept injection in subjects with exudative age-related macular degeneration, previously treated with ranibizumab or bevacizumab: 6-month interim analysis. Br J Ophthalmol. 2014;98(Suppl 1):i22-i27.

23. Kawashima Y, Oishi A, Tsujikawa A, et al. Effects of aflibercept for ranibizumab-resistant neovascular age-related macular degeneration and polypoidal choroidal vasculopathy. Graefes Arch Clin Exp Ophthalmol. 2015;253(9):1471-1477.

24. Chang AA, Broadhead GK, Hong T, et al. Intravitreal aflibercept for treatment-resistant neovascular age-related macular degeneration: 12-month safety and efficacy outcomes. Ophthalmic Res. 2015;55(2): 84-90.

25. Mantel I, Gianniou C, Dirani A. Conversion to aflibercept therapy versus continuing with ranibizumab therapy for neovascular age-related macular degeneration dependent on monthly ranibizumab treatment. Retina. 2016;36(1):53-58.

26. Ogura Y, Terasaki H, Gomi F, et al. Efficacy and safety of intravitreal aflibercept injection in wet age-related macular degeneration: outcomes in the Japanese subgroup of the VIEW 2 study. Br J Ophthalmol. 2015; 99(1):92-97. 
27. Gupta OP, Shienbaum G, Patel AH, Fecarotta C, Kaiser RS, Regillo CD. A treat and extend regimen using ranibizumab for neovascular age-related macular degeneration clinical and economic impact. Ophthalmology. 2010;117(11):2134-2140.

28. Wykoff CC, Croft DE, Brown DM, et al. Prospective trial of treatand-extend versus monthly dosing for neovascular age-related macular degeneration: TREX-AMD 1-year results. Ophthalmology. 2015;122(12): 2514-2522.

29. Brown DM, Chen E, Mariani A, Major JC Jr; SAVE Study Group Super-dose anti-VEGF (SAVE) trial: $2.0 \mathrm{mg}$ intravitreal ranibizumab for recalcitrant neovascular macular degeneration-primary end point. Ophthalmology. 2013;120(2):349-354.

30. Hikichi T, Higuchi M, Matsushita T, et al. Results of 2 years of treatment with as-needed ranibizumab reinjection for polypoidal choroidal vasculopathy. Br J Ophthalmol. 2013;97(5):617-621.

31. Oishi A, Tsujikawa A, Yamashiro K, et al. One-year result of aflibercept treatment on age-related macular degeneration and predictive factors for visual outcome. Am J Ophthalmol. 2015;159(5): 853-860.e851.
32. Yamamoto A, Okada AA, Kano M, et al. One-year results of intravitreal aflibercept for polypoidal choroidal vasculopathy. Ophthalmology. 2015; 122(9):1866-1872.

33. Maruko I, Iida T, Saito M, Nagayama D, Saito K. Clinical characteristics of exudative age-related macular degeneration in Japanese patients. Am J Ophthalmol. 2007;144:15-22.

34. Parodi MB, Iacono $\mathrm{P}$, Papayannis $\mathrm{A}$, et al. Intravitreal ranibizumab for pigment epithelium detachment with subfoveal occult choroidal neovascularization: a prospective 24-month case series. Am J Ophthalmol. 2013;155(1):103-108.e102.

35. Yuzawa M, Mori R, Kawamura A. The origins of polypoidal choroidal vasculopathy. Br J Ophthalmol. 2005;89(5):602-607.

36. Terasaki H, Miyake Y, Suzuki T, Nakamura M, Nagasaka T. Polypoidal choroidal vasculopathy treated with macular translocation: clinical pathological correlation. Br J Ophthalmol. 2002;86(3):321-327.

37. Kim JH, Kang SW, Kim TH, Kim SJ, Ahn J. Structure of polypoidal choroidal vasculopathy studied by colocalization between tomographic and angiographic lesions. Am J Ophthalmol. 2013;156(5): 974-980.e972.
Clinical Ophthalmology

\section{Publish your work in this journal}

Clinical Ophthalmology is an international, peer-reviewed journal covering all subspecialties within ophthalmology. Key topics include: Optometry; Visual science; Pharmacology and drug therapy in eye diseases; Basic Sciences; Primary and Secondary eye care; Patient Safety and Quality of Care Improvements. This journal is indexed on

Submit your manuscript here: http://www.dovepress.com/clinical-ophthalmology-journal

\section{Dovepress}

PubMed Central and CAS, and is the official journal of The Society of Clinical Ophthalmology (SCO). The manuscript management system is completely online and includes a very quick and fair peer-review system, which is all easy to use. Visit http://www.dovepress.com/ testimonials.php to read real quotes from published authors. 